\title{
Probabilistic machine learning for the evaluation of presurgical language dominance
}

\author{
Tomer Gazit, PhD, ${ }^{1}$ Fani Andelman, PhD, ${ }^{2}$ Yifat Glikmann-Johnston, PhD, ${ }^{1}$ Tal Gonen, MA, ${ }^{1,6}$ \\ Aliya Solski, MA, ${ }^{1}$ Irit Shapira-Lichter, PhD, ${ }^{1,3}$ Moran Ovadia, BA, ${ }^{1}$ Svetlana Kipervasser, MD, ${ }^{2-4}$ \\ Miriam Y. Neufeld, MD, , Itzhak Fried, MD, PhD, ${ }^{2,4,5,7}$ Talma Hendler, MD, PhD, ,1,4,6,7 and \\ Daniella Perry, $\mathrm{PhD}^{1}$
}

${ }^{1}$ Functional Brain Center, ${ }^{2}$ Functional Neurosurgery Unit, Department of Neurosurgery and ${ }^{3}$ Department of Neurology, Tel Aviv Souraksy Medical Center; ${ }^{6}$ School of Psychological Sciences and ${ }^{7}$ Sagol School of Neuroscience, ${ }^{4}$ Faculty of Medicine, Tel Aviv University, Tel Aviv, Israel; and ${ }^{5}$ Department of Neurosurgery, David Geffen School of Medicine and Semel Institute for Neuroscience and Human Behavior, University of California, Los Angeles, California

\begin{abstract}
OBJECTIVE Providing a reliable assessment of language lateralization is an important task to be performed prior to neurosurgery in patients with epilepsy. Over the last decade, functional MRI (fMRI) has emerged as a useful noninvasive tool for language lateralization, supplementing or replacing traditional invasive methods. In standard practice, fMRIbased language lateralization is assessed qualitatively by visual inspection of fMRI maps at a specific chosen activation threshold. The purpose of this study was to develop and evaluate a new computational technique for providing the probability of each patient to be left, right, or bilateral dominant in language processing.

METHODS In 76 patients with epilepsy, a language lateralization index was calculated using the verb-generation fMRI task over a wide range of activation thresholds (from a permissive threshold, analyzing all brain regions, to a harsh threshold, analyzing only the strongest activations). The data were classified using a probabilistic logistic regression method.

RESULTS Concordant results between fMRI and Wada lateralization were observed in $89 \%$ of patients. Bilateral and right-dominant groups showed similar fMRI lateralization patterns differentiating them from the left-dominant group but still allowing classification in $82 \%$ of patients.
\end{abstract}

CONCLUSIONS These findings present the utility of a semi-supervised probabilistic learning approach for presurgical language-dominance mapping, which may be extended to other cognitive domains such as memory and attention.

http://thejns.org/doi/abs/10.3171/2015.7.JNS142568

KEY WORDS fMRI; epilepsy; language lateralization; semi-supervised; Wada; logistic regression; functional neurosurgery

A CRUCIAL aspect of neurosurgery within or close to eloquent cortex is the preoperative identification of brain regions involved in a number of cognitive, sensory, or motor tasks. In the presurgical assessment of language, both the localization ${ }^{38}$ and lateralization ${ }^{28,32}$ of functional areas are essential for the prevention of postoperative deficits such as aphasia and dysarthria. More specifically, assessing language lateralization in patients with epilepsy is challenging and complex because of their atypical language network. Language structure among this group tends to be more variable, ${ }^{40}$ less lateralized, ${ }^{11}$ and more subject to plasticity effects ${ }^{21}$ as compared with a healthy population.
Until recently, the gold standard procedure for language lateralization has been the Wada test.7,42 This test determines language and memory lateralization of the cerebral function by suppressing cortical activity in one of the hemispheres, while testing the patient's performance using the other. The test involves the unilateral intra-carotid artery injection of a short-acting barbiturate (amobarbital) that immediately suspends several ipsilateral brain functions, allowing for 5-10 minutes of language or memory tests of the contralateral hemisphere. The Wada test findings represent a categorical classification of left, right, or bilateral hemispheric dominance of language or memory function. However, this invasive procedure has potential clinical

ABBREVIATIONS EPI = echo-planar imaging; fMRI = functional MRI; LDP = language dominance probability; LI = lateralization index; SPGR = spoiled gradient; TASMC = Tel Aviv Sourasky Medical Center. 
complications, such as bleeding ${ }^{20}$ and infection, ${ }^{18}$ and usually involves significant patient discomfort. Moreover, the interpretation of the Wada results may be problematic, as there is no standard procedure for testing language and memory, and in a minority of patients it can produce false lateralization. ${ }^{27}$ Thus, the validity of the Wada examination is still a matter of debate. ${ }^{27,30}$

Due to these issues, the clinical use of noninvasive imaging methods for language lateralization, such as functional MRI (fMRI), is becoming increasingly popular, particularly for patients with epilepsy.,2,20 The common practice of presurgical language mapping involves neuropsychological tests, clinical evaluation, and a qualitative examination of fMRI activations. However, over the years multiple quantitative fMRI-based lateralization measures have been proposed. In its most basic form, a lateralization index (LI) is a value between 0 and 1 detailing the relation of activity between homolog clusters from both the left and right hemispheres. ${ }^{2,17,28,31,34,36,44}$ Since neurosurgeons are typically interested in classifying patients as having left, right, or bilateral language lateralization, classification algorithms can be of much assistance. Traditionally, these methods used an activation cutoff threshold to classify patients into laterality groups, ${ }^{22}$ but this strict classification can be risky, as misclassifications are unavoidable. ${ }^{1}$ Instead, providing the clinical team with language processing lateralization probabilities could be of more benefit. Such a task calls for the utilization of probabilistic machine-learning algorithms, a growing field with multiple applications in medicine, ${ }^{9}$ ranging from the diagnosis of skin lesions ${ }^{19}$ to predicting pneumonia mortality. ${ }^{14}$ Specifically, the task of the algorithm used in this study was to analyze and learn the fMRI-based LI patterns of a large group of patients so that it will be able to probabilistically classify new patients as belonging to different laterality groups (left, bilateral, or right) without the need to perform an invasive Wada procedure for language testing. Moreover, we evaluated various approaches for fMRI lateralization analysis to improve upon common practice; this included establishing optimal activation thresholds and stimuli modality (auditory or visual), assessing the need for invasive validation, and determining the most suitable brain regions for analysis. Finally, additional nonimaging variables, such as hand dominance, neuropsychological testing, age of epilepsy onset, and lesion side were combined with the imaging data to test for the ideal data combination for lateralization classification.

\section{Methods \\ Patients}

The patient group for this study consisted of 76 patients suffering from intractable epilepsy (39 females and 37 males), 44 of whom were right handed (see Table 1 for demographic characteristics). All patients were candidates for surgery and were referred to the functional brain center at the Tel Aviv Sourasky Medical Center (TASMC) for fMRI for presurgical evaluation for the localization and lateralization of language-related functional activity. In addition to fMRI evaluation, a Wada examination for language and memory lateralization was performed on 28 of these patients, all lead by the same neuropsychologist from our center. Using the Wada test, it was determined that 17 patients had left hemispherical language dominance, 5 had bilateral, and 6 had right dominance. The results obtained in this group of 28 patients were used for the initial probabilistic classification of group LI distributions, while testing was performed in the remaining patients to refine these probabilities in a semi-supervised machine-learning procedure (see Machine Learning section). Neuropsychological tests were performed on 50 of the 76 patients (Table 2). This study was approved by the institutional review board of the TASMC.

\section{The fMRI Protocol}

Functional MRI examinations included 2 verb-generation tasks (auditory and visual), during which patients were asked to covertly generate a verb in response to various nouns being presented. The verb-generation task has been found to be reliable in prompting repeated activation of language areas in many fMRI studies ${ }^{10,15,45}$ and has been widely studied as part of a presurgical mapping protocol. ${ }^{35,36}$ Additionally, in comparison with alternative fMRI paradigms, the verb-generation task has been shown to be the task most highly correlated with Wada laterality results. ${ }^{36}$ Twenty-one common Hebrew nouns were presented in blocks of 7 nouns, with each block requiring 20-22 seconds. Each of the 7 nouns in a block was presented for $500 \mathrm{msec}$, with an interstimulus interval of 2-3 seconds. Rest periods of 20-30 seconds were provided between the blocks, and the entire test lasted 204 seconds. For the auditory task patients listened to a recording of nouns using a headset. For the visual task a set of pictures depicting objects were shown to the patient on a computer screen (with a white background).

\section{Image Acquisition and Preprocessing}

Brain scanning was performed with 2 General Electric scanners: a 1.5-T scanner (for 35 patients tested before June 2008) and a 3-T scanner (for 41 patients examined after June 2008). Using the 3-T scanner, fMRI scans were obtained with a gradient echo-planar imaging (EPI) sequence of functional T2*-weighted images (TR 3000 msec, TE $35 \mathrm{msec}$, flip angle $90^{\circ}$, FOV $20 \times 20 \mathrm{~cm}$, matrix $64 \times 64 \mathrm{~mm}$ ) divided into 39 axial slices (thickness 3 $\mathrm{mm}$, gap $0 \mathrm{~mm}$ ). Anatomical 3D spoiled gradient (SPGR) echo sequences were obtained with high-resolution 1-mm slices. For the 1.5-T scanner, fMRI scans were acquired with a gradient EPI sequence of functional T2*-weighted images (TR $3000 \mathrm{msec}$, TE $55 \mathrm{msec}$, flip angle $90^{\circ}$, FOV $24 \times 24 \mathrm{~cm}$, matrix $80 \times 80 \mathrm{~mm}$ ) divided into axial slices (thickness $3 \mathrm{~mm}$, gap $0 \mathrm{~mm}$ ). Anatomical 3D SPGR echo sequences were obtained with high-resolution 1-mm slices.

Initial data analysis was performed using SPM5 software (http://www.fil.ion.ucl.ac.uk/spm). Preprocessing included slice timing correction, 3D motion correction, and co-registration with the anatomical image. The data were spatially smoothed with an 8-mm full-width at half-maximum kernel. The first 6 functional volumes, prior to signal stabilization, were excluded from the analysis. Functional 
TABLE 1. Demographic and clinical characteristics of patients

\begin{tabular}{|c|c|c|c|c|c|c|c|c|}
\hline $\begin{array}{l}\text { Case } \\
\text { No. }\end{array}$ & $\begin{array}{l}\text { Sex, Age } \\
\text { (yrs) }\end{array}$ & Handedness & $\begin{array}{l}\text { Age at Seizure } \\
\text { Onset (yrs) }\end{array}$ & EEG Localization & $\begin{array}{c}\text { Lesion } \\
\text { (MRI/pathology) }\end{array}$ & Medication & $\begin{array}{c}\text { Wada } \\
\text { Test }\end{array}$ & $\begin{array}{l}\text { fMRI } \\
\text { Date }\end{array}$ \\
\hline 1 & $F, 24$ & Rt & 2 & Lt T & Lt MTS & TPX & $\mathrm{Lt}$ & 11.11 \\
\hline 2 & M, 33 & $\mathrm{Lt}$ & 4 & Lt FT & Lt MTS & TPX & Lt & 07.02 \\
\hline 3 & $F, 26$ & $\mathrm{Lt}$ & 19 & Lt T & $\mathrm{NL}$ & TPX & $\mathrm{Lt}$ & 09.05 \\
\hline 4 & $F, 43$ & Rt & 2 & Lt T & Lt MTS & TPX & $\mathrm{Lt}$ & 01.04 \\
\hline 5 & $F, 18$ & $\mathrm{Lt}$ & 11 & Rt T & Rt GA & TPX, LTG & $\mathrm{Lt}$ & 05.08 \\
\hline 6 & $\mathrm{M}, 14$ & $\mathrm{Lt}$ & 0.5 & $\mathrm{~N}$ & Py & VPA, ZNS & $\mathrm{Lt}$ & 07.04 \\
\hline 7 & M, 29 & $\mathrm{Lt}$ & 11 & Rt FT & Rt MTS & LTG & $\mathrm{Lt}$ & 03.09 \\
\hline 8 & $F, 33$ & $\mathrm{Rt}$ & 16 & Rt T & $\mathrm{NL}$ & LTG & $\mathrm{Lt}$ & 05.10 \\
\hline 9 & $F, 32$ & Rt & 28 & Rt T & Rt T Dy & LEV & $\mathrm{Lt}$ & 01.09 \\
\hline 10 & $F, 18$ & $\mathrm{Rt}$ & 14 & Lt T & Lt T DNET & TPX & $\mathrm{Lt}$ & 11.10 \\
\hline 11 & M, 26 & $\mathrm{Rt}$ & 12 & $\mathrm{~N}$ & Lt T GI & VTA, OXC & $\mathrm{Lt}$ & 09.06 \\
\hline 12 & $F, 24$ & $\mathrm{Lt}$ & 4.5 & Rt F & RT T SOL & CBZ, TPX & $\mathrm{Lt}$ & 10.07 \\
\hline 13 & $\mathrm{M}, 20$ & $\mathrm{Rt}$ & 8 & $\mathrm{~N}$ & $\mathrm{NL}$ & LEV, CBZ & $\mathrm{Lt}$ & 02.07 \\
\hline 14 & $M, 16$ & $\mathrm{Rt}$ & 14 & $\mathrm{~N}$ & Lt MTS & LTG & $\mathrm{Lt}$ & 01.08 \\
\hline 15 & $F, 41$ & $\mathrm{Rt}$ & 28 & Lt T & $\mathrm{NL}$ & CBZ & $\mathrm{Lt}$ & 11.06 \\
\hline 16 & M, 24 & Rt & 20 & Lt T & Lt MTS & $\mathrm{N}$ & $\mathrm{Lt}$ & 07.08 \\
\hline 17 & $\mathrm{~F}, 28$ & $\mathrm{Rt}$ & 2 & Rt T & $\mathrm{NL}$ & OXC, LTG, CLB, TPX & Lt & 05.06 \\
\hline 18 & $F, 16$ & $\mathrm{Lt}$ & 1 & $\mathrm{~N}$ & Lt FT AVM & CBZ, TPX & Bilat & 06.05 \\
\hline 19 & $\mathrm{M}, 30$ & $\mathrm{Rt}$ & 17 & Rt FT & Rt TAA & VPA, ZNS & Bilat & 02.03 \\
\hline 20 & M, 33 & Rt & 11 & Lt FT & $\mathrm{NL}$ & CBZ, CLB, LEV & Bilat & 11.06 \\
\hline 21 & $M, 63$ & $M$ & 49 & Lt T & Bilat MTS & LEV & Bilat & 03.12 \\
\hline 22 & M, 43 & Rt & 8 & Rt T & Rt MTS & LTG, CBZ, CLB & Bilat & 11.12 \\
\hline 23 & $\mathrm{~F}, 34$ & $\mathrm{Lt}$ & 9 & Rt T & NL & CBZ, LEV & Rt & 08.05 \\
\hline 24 & $F, 33$ & $\mathrm{Rt}$ & 18 & $\mathrm{~N}$ & RtPSOL & OXC, TPX & $\mathrm{Rt}$ & 09.06 \\
\hline 25 & $F, 26$ & $\mathrm{Lt}$ & 9 & $\mathrm{~N}$ & Lt MTS & LTG & $\mathrm{Rt}$ & 08.02 \\
\hline 26 & M, 25 & $\mathrm{Lt}$ & 0.5 & $\mathrm{LtT}$ & Lt MTS & CBZ, OXC, TPX & Rt & 09.06 \\
\hline 27 & $\mathrm{~F}, 34$ & M & 8 & Rt FT & Rt MTS & VPA, LEV, OXC & $\mathrm{Rt}$ & 12.06 \\
\hline 28 & $F, 13$ & $\mathrm{Lt}$ & 6 & Lt FT & Lt T GI & CBZ, LTG & $\mathrm{Rt}$ & 02.04 \\
\hline 29 & $F, 35$ & $\mathrm{Lt}$ & 14 & $\mathrm{~N}$ & Rt MTS & LEV, TPX, CBZ & $N P$ & 03.09 \\
\hline 30 & $\mathrm{~F}, 40$ & $\mathrm{Lt}$ & 40 & $\mathrm{~N}$ & Rt T As & PTH & NP & 03.09 \\
\hline 31 & M, 27 & $\mathrm{Lt}$ & 3 & Lt FT & $\mathrm{NL}$ & LEV, LTG & NP & 08.09 \\
\hline 32 & M, 42 & $\mathrm{Lt}$ & 18 & Lt FT & Lt MTS & VPA & $N P$ & 10.09 \\
\hline 33 & $M, 52$ & $\mathrm{Lt}$ & 45 & $\mathrm{~N}$ & Lt FTP AVM & CBZ, LTG, TPX & NP & 02.08 \\
\hline 34 & $\mathrm{M}, 14$ & $\mathrm{Lt}$ & 8 & $N$ & $\mathrm{Lt} F \mathrm{Hm}$ & $\mathrm{N}$ & NP & 12.08 \\
\hline 35 & $M, 37$ & $\mathrm{Lt}$ & 32 & $\mathrm{~N}$ & Rt T Ca & CBZ & $N P$ & 10.06 \\
\hline 36 & $F, 33$ & $\mathrm{Lt}$ & 16 & Lt T & $\mathrm{NL}$ & $\mathrm{CBZ}, \mathrm{GBP}$ & $\mathrm{NP}$ & 01.07 \\
\hline 37 & $\mathrm{M}, 51$ & $\mathrm{Lt}$ & 51 & $\mathrm{~N}$ & Rt MTS & $\mathrm{N}$ & $\mathrm{NP}$ & 05.07 \\
\hline 38 & $\mathrm{~F}, 11$ & $\mathrm{Rt}$ & 11 & Rt T & Rt T SOL & $\mathrm{CBZ}$ & NP & 06.07 \\
\hline 39 & $F, 24$ & $\mathrm{Lt}$ & 8 & $\mathrm{~N}$ & Lt T Ol & CBZ, TPX & NP & 06.07 \\
\hline 40 & $\mathrm{~F}, 11$ & $\mathrm{Lt}$ & 11 & $\mathrm{~N}$ & Rt FP DNET & PTH & $\mathrm{NP}$ & 12.07 \\
\hline 41 & $F, 56$ & $\mathrm{Lt}$ & 47 & $N$ & Lt O Ol & CBZ, LEV & NP & 12.07 \\
\hline 42 & $\mathrm{M}, 12$ & $\mathrm{Rt}$ & 8 & $\mathrm{~N}$ & $\mathrm{NL}$ & VPA & $N P$ & 09.11 \\
\hline 43 & M, 46 & Rt & 45 & $\mathrm{~N}$ & LtF SOL & CBZ & $N P$ & 09.11 \\
\hline 44 & $\mathrm{M}, 32$ & Rt & 32 & $\mathrm{~N}$ & LtF SOL & $\mathrm{N}$ & $N P$ & 02.01 \\
\hline 45 & $F, 26$ & $\mathrm{Rt}$ & 25 & $\mathrm{~N}$ & LtF Ca & $\mathrm{CBZ}$ & $N P$ & 08.11 \\
\hline 46 & M, 25 & $\mathrm{Rt}$ & 11 & Lt T & Lt T & LEV, CBZ & $N P$ & 08.11 \\
\hline 47 & $\mathrm{M}, 19$ & $\mathrm{Rt}$ & 0.8 & Lt F & Lt FT GA & LEV, OXC, TPX, CLB & NP & 08.11 \\
\hline 48 & $\mathrm{M}, 21$ & $\mathrm{Rt}$ & 14 & Rt T & Rt MTS & LEV, VPA, GBP & NP & 07.11 \\
\hline
\end{tabular}


TABLE 1. Demographic and clinical characteristics of patients (continued)

\begin{tabular}{|c|c|c|c|c|c|c|c|c|}
\hline $\begin{array}{l}\text { Case } \\
\text { No. }\end{array}$ & $\begin{array}{l}\text { Sex, Age } \\
\text { (yrs) }\end{array}$ & Handedness & $\begin{array}{l}\text { Age at Seizure } \\
\text { Onset (yrs) }\end{array}$ & EEG Localization & $\begin{array}{c}\text { Lesion } \\
\text { (MRI/pathology) }\end{array}$ & Medication & $\begin{array}{c}\text { Wada } \\
\text { Test }\end{array}$ & $\begin{array}{l}\text { fMRI } \\
\text { Date }\end{array}$ \\
\hline 49 & $F, 34$ & $\mathrm{Rt}$ & 31 & $\mathrm{~N}$ & Rt F As & CLB, GBP & NP & 07.00 \\
\hline 50 & M, 12 & $\mathrm{Rt}$ & 2 & Lt T & LtPAs & LTG & NP & 10.02 \\
\hline 51 & $F, 32$ & Bilat & 25 & $\mathrm{~N}$ & $\mathrm{NL}$ & CLB, TPX & NP & 05.11 \\
\hline 52 & M, 24 & $\mathrm{Rt}$ & 24 & $\mathrm{~N}$ & Lt P Ol & PTH, VPA & NP & 05.11 \\
\hline 53 & $\mathrm{M}, 48$ & $\mathrm{Rt}$ & 48 & $\mathrm{~N}$ & Lt TOI & PTH, CBZ & NP & 04.11 \\
\hline 54 & $F, 8$ & Lt & 0 & $\mathrm{~N}$ & NL & OXC, LEV, VPA & NP & 04.11 \\
\hline 55 & $F, 18$ & Rt & 17 & RtF & RtPAs & CBZ & NP & 03.11 \\
\hline 56 & M, 26 & Rt & 5 & $\mathrm{~N}$ & Lt T + Rt F Dy & LEV, CBZI & NP & 03.11 \\
\hline 57 & $\mathrm{M}, 10$ & $\mathrm{Lt}$ & 6 & $\mathrm{Lt}+\mathrm{Rt} F$ & Lt MTS & LCM & NP & 02.11 \\
\hline 58 & $F, 29$ & Rt & 12 & Lt TP & LtP GI & LEV & NP & 02.11 \\
\hline 59 & $F, 37$ & $\mathrm{Rt}$ & 36 & $\mathrm{~N}$ & Lt F Ca & VPA & $\mathrm{NP}$ & 01.11 \\
\hline 60 & $F, 35$ & $\mathrm{Lt}$ & 35 & $\mathrm{~N}$ & Rt F SOL & VPA & $\mathrm{NP}$ & 01.11 \\
\hline 61 & $F, 11$ & Rt & 10 & $\mathrm{~N}$ & Lt F DNET & $\mathrm{N}$ & NP & 12.10 \\
\hline 62 & $F, 16$ & Rt & 13 & $\mathrm{~N}$ & Lt F Ca & CBZ & NP & 10.10 \\
\hline 63 & $M, 63$ & Rt & 63 & $\mathrm{~N}$ & Lt P Ca & $\mathrm{N}$ & NP & 10.10 \\
\hline 64 & M, 29 & $\mathrm{Rt}$ & 12 & Bilat F & $\mathrm{NL}$ & VPA, CLB, OXC & NP & 10.10 \\
\hline 65 & $F, 12$ & $\mathrm{Lt}$ & 12 & Rt T & Rt T DNET & LTG & NP & 09.10 \\
\hline 66 & $\mathrm{M}, 31$ & $\mathrm{Rt}$ & 7 & Rt TP & NL & OXC, LEV & NP & 09.10 \\
\hline 67 & $\mathrm{M}, 30$ & $\mathrm{Lt}$ & 15 & Lt TP & $\mathrm{NL}$ & GBP, OXC, CLB & $\mathrm{NP}$ & 08.10 \\
\hline 68 & $M, 33$ & Lt & 22 & $\mathrm{~N}$ & Rt FT Dy & VPA, LEV & NP & 08.10 \\
\hline 69 & M, 23 & Bilat & 4 & Lt & Lt PO SOL & LEV, CBZ, TPX & NP & 06.10 \\
\hline 70 & $\mathrm{~F}, 27$ & Rt & 8 & Rt T & Rt T SOL & $\mathrm{N}$ & NP & 04.10 \\
\hline 71 & $\mathrm{M}, 19$ & $\mathrm{Rt}$ & 0 & $\mathrm{~N}$ & $\mathrm{NL}$ & LEV, TPX & NP & 04.10 \\
\hline 72 & $F, 22$ & Rt & 10 & Lt T & Lt T Dy & LTG, CLB & NP & 07.04 \\
\hline 73 & $F, 43$ & $\mathrm{Rt}$ & 13 & Lt FT & Lt T DNET & TPX & NP & 01.04 \\
\hline 74 & $F, 38$ & $\mathrm{Rt}$ & 28 & $\mathrm{~N}$ & Rt F SOL & CBZ & NP & 02.10 \\
\hline 75 & $M, 16$ & $\mathrm{Rt}$ & 3 & $\mathrm{~N}$ & Lt FP SOL & $\mathrm{PR}, \mathrm{OXC}$ & NP & 01.10 \\
\hline 76 & $F, 29$ & $\mathrm{Rt}$ & 26 & $\mathrm{~N}$ & Rt P Ca & CBZ & NP & 01.10 \\
\hline
\end{tabular}

$\mathrm{AA}=$ anaplastic astrocytoma; $\mathrm{As}=$ astrocytoma; $\mathrm{AVM}=$ arteriovenous malformation; $\mathrm{Ca}=$ cavernoma; $\mathrm{CBZ}=$ carbamazepine; $\mathrm{CLB}=$ clobazam; $\mathrm{DNET}=$ dysembryoplastic neuroepithelial tumor; $\mathrm{Dy}=$ dysplasia; $E E G=$ electroencephalogram; $F=$ frontal; $F P=$ frontoparietal; $F T=$ frontotemporal; $F T P=$ frontotemporoparietal; $G A=$ general atrophy; $\mathrm{GBP}=$ gabapentin; $\mathrm{GI}$ = gliosis; $\mathrm{Hm}$ = hematoma; $\mathrm{LCM}=$ lacosamide; $\mathrm{LEV}=$ levetiracetam; $\mathrm{LTG}=$ lamotrigine; $\mathrm{M}=$ mixed; $M T S=$ mesial temporal sclerosis; $\mathrm{N}=$ none; $\mathrm{NL}=$ no lesion; $\mathrm{NP}=$ not performed; $\mathrm{O}=$ occipital; $\mathrm{OI}=$ oligodendroglioma; $\mathrm{OXC}=$ oxcarbazepine; $\mathrm{P}=$ parietal; $\mathrm{PO}=$ parietooccipital; $\mathrm{PR}=$ primidone; PTH = phenytoin; $\mathrm{Py}=$ porencephaly; $\mathrm{SOL}=$ space-occupying lesion; $\mathrm{T}=$ temporal; $\mathrm{TP}=$ temporoparietal; $\mathrm{TPX}=$ topiramate; $\mathrm{VPA}=$ valproic acid; $\mathrm{ZNS}=$ zonisamide .

EPI data were automatically aligned and co-registered with 3D anatomical data and manually corrected as necessary. Functional analysis was performed using a general linear model in which the activation blocks were defined as the predictors. To account for hemodynamic responses, predictors were shifted by 6 seconds.

\section{Modalities and Localization}

Language lateralization was assessed for both the visual and auditory verb-generation fMRI tasks and their conjunction. The selection of brain regions to be considered when assessing language lateralization is still debated in the literature, with results pointing to both a whole-brain approach and Broca's area alone as optimal modes of assessment. ${ }^{16,23,28}$ To calculate lateralization we employed both a whole-brain ${ }^{6,8,23}$ and region-specific approach, using Broca's area, the known primary production region, as a region of interest. For the whole-brain approach, 3 re- gions were removed from analysis, as they appeared to be less specific for language processing and could potentially skew calculations by adding noise. These areas included the occipital lobe, the cerebellum, and the interhemispheric fissure. ${ }^{2,6}$ For the analysis of Broca's Area, anatomical and functional scans were normalized to the Montreal Neurological Institute template (using SPM [statistical parametric mapping]), and functional maps were masked at Brodmann areas 44 and 45 using the WFU-PickAtlas SPM toolbox. ${ }^{29}$ As normalization to an anatomical template was required for masking with Broca's area, 7 of the 28 Wada cases were excluded from this analysis because substantial lesions prevented adequate normalization. The remaining 21 patients, including 12 left-, 4 bilateral-, and 5 right-dominance Wada patients were analyzed. The performance of the whole-brain approach in terms of compatibility with Wada results was compared with that of the $\mathrm{Br}-$ oca's area analysis, and the performance of the algorithm 
TABLE 2. Neuropsychological and clinical data relevant to lateralization*

\begin{tabular}{|c|c|c|c|c|c|c|c|c|c|c|}
\hline \multirow{2}{*}{$\begin{array}{l}\text { Case } \\
\text { No. }\end{array}$} & \multirow[b]{2}{*}{ Handedness } & \multirow{2}{*}{$\begin{array}{l}\text { Age at Epilepsy } \\
\text { Onset (yrs) }\end{array}$} & \multirow{2}{*}{$\begin{array}{l}\text { Duration of } \\
\text { Epilepsy }\end{array}$} & \multirow{2}{*}{$\begin{array}{l}\text { Expected Focus } \\
\text { Side }\end{array}$} & \multicolumn{3}{|c|}{ Raw Score } & \multicolumn{3}{|c|}{ Scaled Score on WMS } \\
\hline & & & & & BNT† & COWAT & RAVLT $\ddagger$ & LM II & VPA II & Faces I \\
\hline 1 & Rt & 2 & 22 & Lt & 36 & 12 & 8 & 3 & 9 & 5 \\
\hline 2 & $\mathrm{Lt}$ & 4 & 29 & $\mathrm{Lt}$ & 44 & 10 & 8 & NA & NA & NA \\
\hline 3 & $\mathrm{Lt}$ & 19 & 7 & $\mathrm{Lt}$ & 45 & 10 & 3 & 10 & 3 & 10 \\
\hline 4 & $\mathrm{Rt}$ & 2 & 41 & Lt & 36 & 40 & 7 & 7 & 4 & 10 \\
\hline 5 & $\mathrm{Lt}$ & 11 & 7 & $\mathrm{Rt}$ & 48 & 30 & 9 & NA & 12 & 7 \\
\hline 7 & $\mathrm{Lt}$ & 11 & 18 & $\mathrm{Rt}$ & 44 & 9 & 9 & NA & 9 & 10 \\
\hline 8 & Rt & 16 & 17 & $\mathrm{Rt}$ & 46 & 41 & 6 & 12 & 11 & 9 \\
\hline 9 & $\mathrm{Rt}$ & 28 & 4 & $\mathrm{Rt}$ & 45 & 46 & 11 & 13 & 7 & 10 \\
\hline 10 & $\mathrm{Rt}$ & 14 & 4 & $\mathrm{Lt}$ & 43 & 12 & 8 & 11 & 3 & 7 \\
\hline 11 & $\mathrm{Rt}$ & 12 & 14 & $\mathrm{Lt}$ & 47 & 38 & 13 & 12 & 13 & 10 \\
\hline 12 & $\mathrm{Lt}$ & 4.5 & 19 & $\mathrm{Rt}$ & NA & NA & 12 & 11 & NA & 8 \\
\hline 13 & $\mathrm{Rt}$ & 8 & 12 & UN & 37 & 12 & 6 & 9 & 6 & 9 \\
\hline 14 & $\mathrm{Rt}$ & 14 & 2 & Lt & 44 & 27 & 7 & 10 & NA & NA \\
\hline 15 & Rt & 28 & 13 & $\mathrm{Lt}$ & 48 & 28 & 12 & 11 & 10 & 9 \\
\hline 16 & $\mathrm{Rt}$ & 20 & 4 & $\mathrm{Lt}$ & 46 & 27 & 8 & NA & NA & 10 \\
\hline 17 & $\mathrm{Rt}$ & 2 & 26 & $\mathrm{Rt}$ & 35 & 29 & 10 & 3 & 6 & 6 \\
\hline 18 & $\mathrm{Lt}$ & 1 & 15 & $\mathrm{Lt}$ & 39 & 24 & 7 & 9 & 2 & 7 \\
\hline 19 & $\mathrm{Rt}$ & 17 & 13 & $\mathrm{Rt}$ & 47 & NA & 11 & 17 & NA & NA \\
\hline 20 & $\mathrm{Rt}$ & 11 & 22 & $\mathrm{Lt}$ & 47 & 40 & 10 & 12 & 8 & 8 \\
\hline 21 & $M$ & 49 & 14 & $\mathrm{Lt}$ & 46 & 44 & 4 & 10 & 6 & 7 \\
\hline 22 & $\mathrm{Rt}$ & 8 & 35 & $\mathrm{Rt}$ & 46 & 29 & 6 & 10 & 13 & 8 \\
\hline 23 & $\mathrm{Lt}$ & 9 & 25 & $\mathrm{Rt}$ & 47 & 54 & 11 & 13 & 9 & 12 \\
\hline 24 & $\mathrm{Rt}$ & 18 & 15 & $\mathrm{Rt}$ & 36 & 18 & 5 & 8 & 3 & 6 \\
\hline 25 & $\mathrm{Lt}$ & 9 & 17 & $\mathrm{Lt}$ & 44 & 16 & 12 & NA & NA & NA \\
\hline 26 & $\mathrm{Lt}$ & 0.5 & 24 & $\mathrm{Lt}$ & 44 & 47 & 8 & 3 & 7 & 6 \\
\hline 27 & M & 8 & 26 & $\mathrm{Rt}$ & 41 & 24 & 4 & 5 & 4 & 12 \\
\hline 32 & $\mathrm{Lt}$ & 18 & 24 & $\mathrm{Lt}$ & 47 & 43 & 10 & 12 & 13 & 15 \\
\hline 36 & $\mathrm{Lt}$ & 16 & 17 & $\mathrm{Lt}$ & NA & 55 & 8 & 10 & 7 & 9 \\
\hline 37 & $\mathrm{Lt}$ & 51 & 0 & $\mathrm{Rt}$ & 46 & 25 & 5 & 9 & 6 & 10 \\
\hline 38 & $\mathrm{Rt}$ & 11 & 0 & $\mathrm{Rt}$ & 39 & 27 & 11 & 10 & NA & 8 \\
\hline 42 & $\mathrm{Rt}$ & 8 & 4 & UN & 33 & NA & 11 & NA & NA & NA \\
\hline 44 & $\mathrm{Rt}$ & 32 & 0 & $\mathrm{Lt}$ & 45 & 39 & 8 & 15 & 5 & 7 \\
\hline 46 & $\mathrm{Rt}$ & 11 & 14 & $\mathrm{Lt}$ & 34 & 15 & 9 & NA & NA & NA \\
\hline 47 & $\mathrm{Rt}$ & 0.8 & 18 & $\mathrm{Lt}$ & 6 & 4 & 8 & NA & NA & 5 \\
\hline 49 & $\mathrm{Rt}$ & 31 & 3 & $\mathrm{Rt}$ & 48 & 46 & 7 & 9 & 10 & 10 \\
\hline 51 & $M$ & 25 & 7 & UN & 47 & 35 & 9 & 10 & 13 & 10 \\
\hline 55 & $\mathrm{Rt}$ & 17 & 1 & $\mathrm{Rt}$ & 46 & 26 & 14 & 13 & 10 & 9 \\
\hline 56 & $\mathrm{Rt}$ & 5 & 21 & Bilat & 45 & 28 & 10 & 13 & 11 & 10 \\
\hline 57 & $\mathrm{Lt}$ & 6 & 4 & Lt & 44 & 27 & 9 & 12 & NA & NA \\
\hline 58 & $\mathrm{Rt}$ & 12 & 17 & Lt & 40 & NA & 8 & 7 & 11 & 9 \\
\hline 64 & $\mathrm{Rt}$ & 12 & 17 & Bilat & 47 & 13 & 8 & 14 & NA & 8 \\
\hline 65 & $\mathrm{Lt}$ & 12 & 0 & Bilat & 39 & 28 & 9 & 4 & NA & NA \\
\hline 67 & $\mathrm{Lt}$ & 15 & 15 & Lt & 26 & 34 & 3 & 12 & 3 & 10 \\
\hline 68 & $\mathrm{Lt}$ & 22 & 11 & $\mathrm{Rt}$ & 47 & 39 & 12 & 10 & 13 & 14 \\
\hline 69 & $M$ & 4 & 19 & $\mathrm{Lt}$ & 38 & 11 & 8 & 8 & 9 & 12 \\
\hline 71 & $\mathrm{Rt}$ & 0 & 19 & UN & 44 & 10 & 7 & 4 & 5 & 10 \\
\hline 72 & $\mathrm{Rt}$ & 10 & 12 & Lt & 39 & 52 & 14 & 13 & 11 & 11 \\
\hline
\end{tabular}


TABLE 2. Neuropsychological and clinical data relevant to lateralization* (continued)

\begin{tabular}{|c|c|c|c|c|c|c|c|c|c|c|}
\hline \multirow{2}{*}{$\begin{array}{l}\text { Case } \\
\text { No. }\end{array}$} & \multirow[b]{2}{*}{ Handedness } & \multirow{2}{*}{$\begin{array}{c}\text { Age at Epilepsy } \\
\text { Onset (yrs) }\end{array}$} & \multirow{2}{*}{$\begin{array}{c}\text { Duration of } \\
\text { Epilepsy }\end{array}$} & \multirow{2}{*}{$\begin{array}{l}\text { Expected Focus } \\
\text { Side }\end{array}$} & \multicolumn{3}{|c|}{ Raw Score } & \multicolumn{3}{|c|}{ Scaled Score on WMS } \\
\hline & & & & & BNT† & COWAT & RAVLT $\ddagger$ & LM II & VPA II & Faces I \\
\hline 73 & Rt & 13 & 30 & Lt & 34 & 26 & 11 & 8 & 2 & 6 \\
\hline 74 & Rt & 28 & 10 & $\mathrm{Rt}$ & 47 & 23 & 4 & 12 & 13 & 11 \\
\hline 76 & Rt & 26 & 3 & Rt & 47 & 32 & 10 & 12 & 8 & 10 \\
\hline
\end{tabular}

BNT = Boston Naming Test; COWAT = Controlled Oral Word Association Test; LM II = Logical Memory II; NA = not available; RAVLT = Rey Auditory Verbal Learning Test; UN = unknown; VPA = Verbal Paired Associates; WMS = Wechsler Memory Scale.

* Scaled scores are provided on a scale with a mean value of 10 and SD of 1.5 .

$\dagger$ Raw scores out of 48 .

$\ddagger$ Raw scores out of 15 .

using fMRI of each of the modalities (visual and auditory) was compared with their conjunction in a similar manner.

\section{Lateralization Index Assessment}

\section{Functional MRI Maps at Different Activation Thresholds}

Classically, to produce fMRI activation maps, an LI cutoff threshold must be applied. While choosing a permissive threshold will yield activation in most brain regions, choosing a strict threshold will result in the analysis of only the strongest regions of activation. Hence, a permissive threshold has the potential to include noise from nonrelevant brain regions, but a strict threshold has the potential to be unreliable because it is based on a small number of voxels. As previous studies have chosen to use various thresholds, the threshold problem has become a significant issue that is greatly debated in the literature ${ }^{31}$ To deal with this issue, we calculated the LI for a range of thresholds, from the most permissive to the most strict (Fig. 1), and we performed machine learning to allow the algorithm to choose the best threshold or combination of thresholds for classification. In accordance with Chlebus et al., ${ }^{13}$ the thresholds were chosen on the basis of the activation percentage acquired from all brain voxels. For example, a 10\% threshold results in the analysis of only one-tenth of possible brain voxels (the $10 \%$ with the strongest activation). For the whole-brain analysis, percentage thresholds ranged from $0.1 \%$ to $50 \%$ of all brain volume voxels (91 thresholds in total). Ten representative thresholds in a logarithmic scale-50\%, 25\%, $12.5 \%, 6.3 \%, 3.1 \%, 1.6 \%$, $0.8 \%, 0.4 \%, 0.2 \%$, and $0.1 \%$-were used for further statistical and classification analysis. For the Broca's area analysis, these thresholds were adjusted due to the decrease in total volume analyzed. Percentage thresholds ranged from $4.4 \%$ to $100 \%$ of the total volume for Brodmann areas 44 and 45. Further statistical analysis was also conducted on 10 representative thresholds in log scale: $100 \%, 70 \%, 50 \%$, $35 \%, 25 \%, 17 \%, 12.5 \%, 8.8 \%, 6.3 \%$, and $4.4 \%$. This procedure produced fMRI activation maps for each representative activation threshold. In total, 60 maps were created for each patient: 2 regional approaches (whole brain and Broca's area) $\times 3$ modalities (visual, auditory, and conjunction) $\times 10$ representative thresholds.

\section{Lateralization Index Calculation}

Next, for each of the 60 threshold maps per patient, we calculated the LI using a standard lateralization formula, $L I(\varepsilon)=L_{\varepsilon}-R_{\varepsilon} / L_{\varepsilon}+R_{\varepsilon}$, where $L_{\varepsilon}$ and $R_{\varepsilon}$ represent the sum of activation remaining using a threshold of $\varepsilon$ for the map of the left and right hemisphere, respectively. This calculation produced a value between -1 and 1 , with complete right or left lateralization represented by LI values of -1 or 1 , respectively, and complete bilateral dominance represented by an LI value of 0 . The LI score was calculated for each of the percentage thresholds across the entire range, with a plot of language lateralization per threshold obtained for each patient.

\section{Machine Learning}

\section{The Algorithm}

The purpose of the current study is to provide each patient's probability of having left, bilateral, or right dominance of language processing. To produce language dominance probabilities (LDPs), we applied logistic regression, a probabilistic machine-learning algorithm (available in a supplementary Appendix, published online only; please refer to the Supplementary Information section at the end of the article). Logistic regression examines the distribution of different groups, in this case using the LI threshold's specific values and additional clinical and neuropsychological measures, to indicate the probability of a novel data set as belonging to each group. ${ }^{9}$ Specifically, the algorithm starts by classifying patients into 2 groups-left and nonleft dominance - and then proceeds to further classify the nonleft group into bilateral or right dominance groups. "Ground truth" classification labeling is provided by the Wada test, which was performed on 28 of the patients included in our study. However, due to this relatively small sample size, the use of these 28 labeled examples may be insufficient for studying the distribution of the different groups, particularly for the bilateral and right groups, which had fewer patients. We thus compared supervised learning to semi-supervised learning. Semi-supervised learning is a relatively new branch of machine learning, allowing the use of nonlabeled data (in this case, patients without Wada lateralization) to improve classification. ${ }^{3,12}$ In this work we applied the semi-supervised logistic regression method introduced by Amini and Gallinari. ${ }^{3}$

\section{Evaluation}

To correctly evaluate the validity of an algorithm, it is customary in machine learning to separate the training group from the test group. When using small study groups, one of the most commonly used methods is the leave-oneout approach. ${ }^{26,41}$ In this approach, the data set of each sub- 


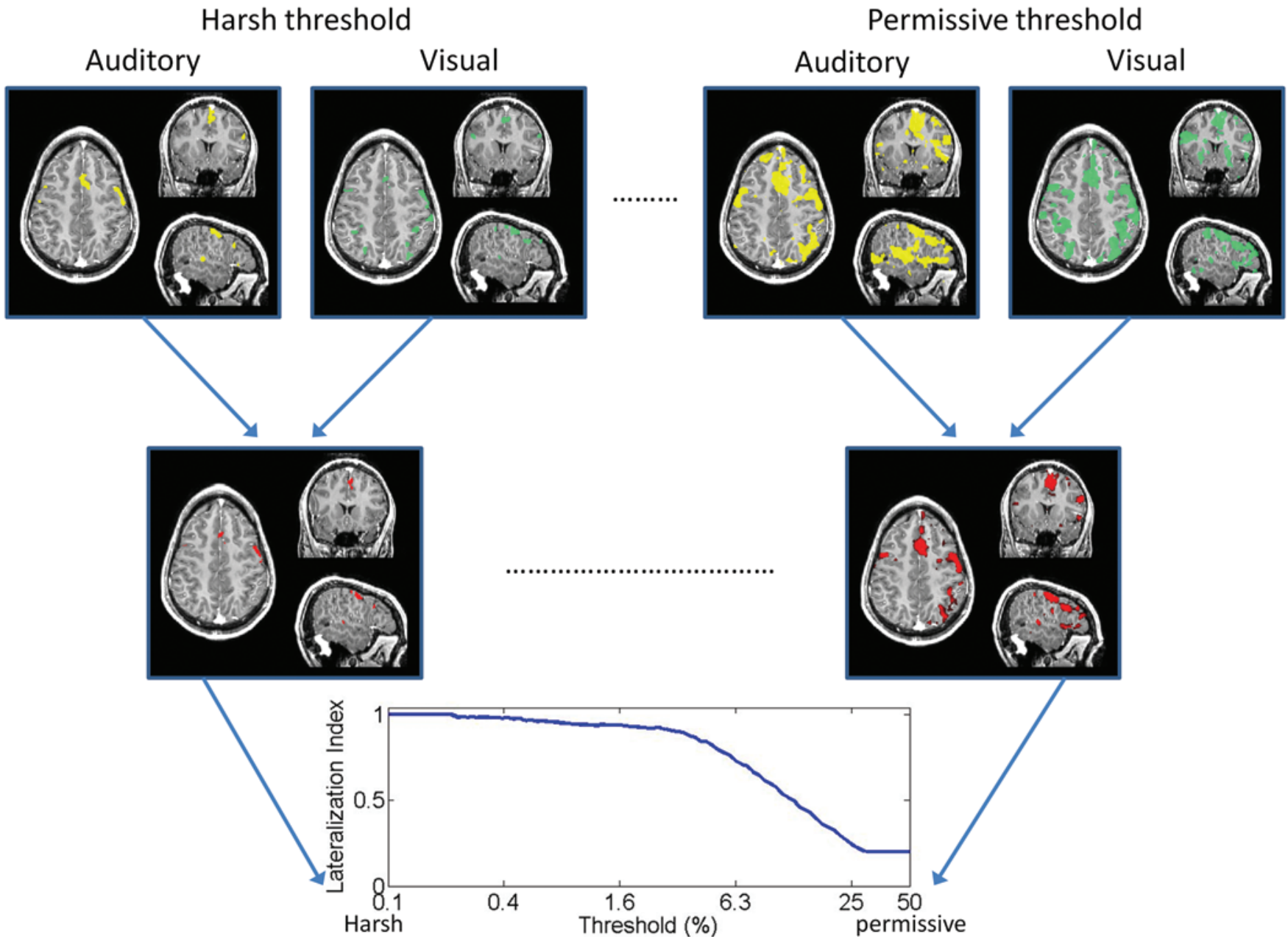

FIG. 1. Scheme of $\mathrm{LI}$ calculation procedure for conjunction analysis using activation maps obtained in an exemplary patient (Case 43). For each of a wide range of activation thresholds, fMRI maps of the auditory (yellow) and visual (green) verb-generation tests are thresholded and masked to frontotemporoparietal regions. The maps are then conjoined (red), and an LI score is calculated, producing a graph of $\mathrm{LI}$ per threshold. Figure is available in color online only.

ject who performed the Wada examination was removed from the data, and the algorithm had to learn from the data of the remaining subjects (training set). Finally, classification of the removed subject, using the parameters learned from the training set, was evaluated against the Wada result (test set). To evaluate the final competence of the lateralization method proposed in this study, we performed the semi-supervised logistic regression algorithm 28 times, each time leaving one of the labeled patients out of the analysis. Thus, the analysis contained 27 labeled patients and 48 unlabeled patients each time it was performed and calculated the patient's probability of belonging to each of the lateralization groups.

\section{Neuropsychological Testing}

Neuropsychological testing was conducted with 50 of the 76 patients. The specific tests to be used were determined by an expert neuropsychologist (F.A.) based on the clinical needs of each patient. Therefore, not all tests were performed for all patients. Overall, the tests included 2 language and 4 memory measures. The language tests included the Hebrew version of the Boston Naming Test ${ }^{25}$ and the Hebrew version of the Controlled Oral Word Association Test. ${ }^{24}$ The memory tests included the Rey Auditory Verbal Learning Test $^{39}$ and 3 subtests from the Wechsler Memory Scale: ${ }^{43}$ Logical Memory II (delayed recall), Verbal Paired Associates II (delayed recall), and Faces I (Table 2). Thirty-three patients, 18 of whom had undergone the Wada test, performed all 6 measures. For the combined analysis, clinical, neuropsychology, and fMRI data were used, with a total of 20 heterogenic features that were fed into the algorithm. The algorithm then calculated the best combination of the data for classification. The features included 6 neuropsychological measures; 10 fMRIbased LIs calculated from 10 thresholds; and clinical data, which consisted of hand dominance, age of epilepsy onset, duration of epilepsy, and the expected hemispheric location of the epileptic foci. In addition, language measures only were administered to an additional group of $12 \mathrm{pa}-$ tients, resulting in a total of 45 patients ( 23 of whom had also undergone a Wada test). In this group, we excluded the 4 memory neuropsychological measures, and thus in- 
cluded only 16 features. In the comparison stage, we thus evaluated whether improved classification can be achieved when feeding the algorithm clinical and neuropsychological data in addition to the fMRI-based LIs.

\section{Comparisons}

Five comparisons were made to evaluate each of the components of the proposed algorithm: 1) single-threshold analysis, feeding the algorithm with LIs of just 1 of the 10 representative thresholds, was compared with multithreshold analysis, feeding the algorithm with LIs of all 10 representative thresholds and allowing it to determine their optimal combination for classification; 2) single-modality-based analysis (visual or auditory) was compared with the conjunction analysis; 3) supervised logistic regression (only Wada-labeled patients) was compared with semi-supervised regression (analyzing both Wada and non-Wada patients); 4) a whole-brain approach was compared with analysis using Broca's area only; and 5) weighting of the clinical and neuropsychological data with the fMRI data was compared with fMRI data alone. All analyses were compared by evaluating the algorithm's LDPs with the lateralization groups determined by the Wada test. For example, if the Wada test produced a left lateralization, then the LDP pointing to left lateralization was taken as the correct probability for classification. Due to the small number of patients in each lateralization group, the nonparametric Wilcoxon test was used to compare between the different analyses. In addition, to evaluate the added benefit of the machine-learning algorithm used in this study over classical methods, we compared our results with the results of a single-threshold and cutoff lateralization method. Janecek et al. ${ }^{22}$ who conducted the most comprehensive study comparing Wada and fMRI (229 patients), used LI cutoffs of +0.25 and -0.25 to classify patients into left versus nonleft and bilateral versus right groups, respectively. Therefore, we applied the method used by Janecek et al. to our data and compared its classification to our results.

\section{Intracarotid Amobarbital (Wada) Procedure}

Patients underwent an intra-carotid artery procedure according to the TASMC routine protocol. ${ }^{4,5}$ Following a vascular anatomy examination, a catheter was placed in the internal carotid artery using a transfemoral approach. None of the patients exhibited evidence of significant cross-flow or anomalous vascularization that could affect the interpretation of the test results. Intra-carotid artery injection of an anesthetic drug (sodium amytal, Brevital, or etomidate) was administered by syringe through the catheter. The hemisphere ipsilateral to the epileptogenic focus was injected first. Language testing was followed by the presentation of 8 objects, beginning with the establishment of contralateral hemiplegia. Language evaluation involved the following 4 tasks: naming 8 objects, reading, following simple commands, and repeating words and sentences. Disruptions during any of these language tasks or the appearance of paraphasia upon recovery were considered signs of language localization in the injected hemisphere.

\section{Results}

\section{Functional Network Patterns}

Functional MRI activations were typically found in regions considered to be part of the language-processing functional network, ${ }^{33}$ consisting of the inferior and middle frontal gyri, the premotor cortex, the supplementary motor area, and the anterior insula (frontal regions), along with the superior temporal sulcus, the supramarginal gyrus, and the angular gyrus (posterior regions). Activations were also found in the cerebellum and regions of the parietal and occipital lobes. The visual verb-generation task produced strong activations in the primary and secondary visual cortex, while the auditory task typically activated the auditory cortex in the superior temporal gyrus and sylvian fissure.

When analyzing the conjunction of the visual and auditory tasks along with the removal of the cerebellum, occipital lobe, and interhemispheric fissure, a more classic language network of activation was observed. However, additional nonspecific regions, such as regions in the parietal lobe and prefrontal cortex, were sometimes observed as well.

\section{Functional MRI Laterality Index of Wada Patients}

Six graphs (3 modalities $\times 2$ regional approaches) of LI per threshold were created for each patient, from a strict analysis of only the strongest $0.1 \%$ activations to a permissive analysis of $50 \%$ of brain regions. The results of the auditory-visual conjunction and whole-brain analysis of 28 Wada patients are shown in Fig. 2. All patients with a Wada result of left lateralization showed a positive LI for the entire threshold range and typically showed an increase in laterality as thresholds became more stringent. All patients with a Wada test result of right lateralization showed a negative LI for the entire threshold range and typically showed a decrease in laterality as thresholds became more stringent. Four of 5 patients with a bilateral lateralization Wada test result showed a negative LI for the entire threshold range, but generally with a weaker lateralization polarity in comparison with right-lateralization patients.

\section{Machine Learning}

Using the leave-one-out approach by combining Wada and non-Wada-labeled data (semi-supervised), conjoining visual and auditory maps, and allowing the algorithm to depict optimal thresholds, we found that all 17 left-Wada patients revealed the highest LDP for left language lateralization (100\% success), 4 of 5 bilateral Wada patients showed the highest LDP for bilateral language lateralization (80\% success), and 4 of 6 right-Wada patients showed the highest LDP for right language lateralization (67\%). The final results and probabilities produced by the algorithm are summarized in Fig. 3.

In the machine-learning algorithm, weights are produced for each threshold in accordance with their significance for classification. The algorithm's final decision regarding the most informative fMRI activation thresholds differed between the classification of left versus nonleft and the classification of bilateral versus right. While the 


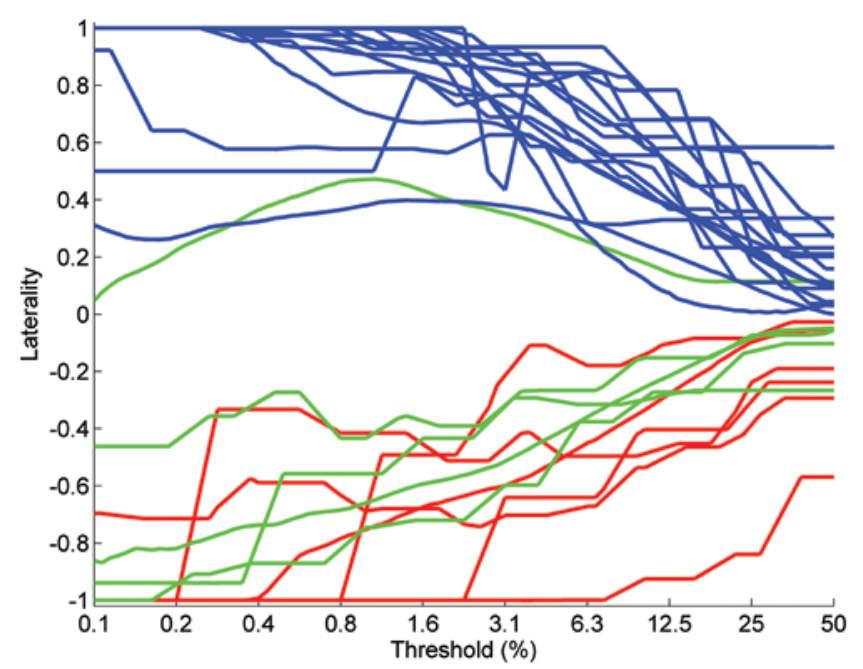

FIG. 2. Graph showing Lls of 28 patients with Wada test results across the different thresholds using the conjunction whole-brain approach. Seventeen left-Wada patients are shown in blue (black), 5 bilateral Wada patients are shown in green (light gray), and 6 right Wada patients are shown in red (gray). Figure is available in color online only.

algorithm found that a strict threshold $(0.1 \%)$ better represented the difference between left and nonleft lateralization, it also found that a more permissive threshold (6.3\%) better represented the difference between bilateral and right lateralization in patients.

\section{Statistical Comparison of Analyses}

To establish the best variables to be considered for lateralization determination in common clinical practice, we compared different possible approaches.

\section{Multithresholds Versus Single Threshold}

The results of 1 multithreshold analysis and 10 singlethreshold analyses were compared. This was performed to evaluate whether a combination of thresholds provided better or worse classification compared with the use of single thresholds. For each Wada-labeled patient (28) and threshold method (11), the leave-one-out logistic regression produced a probability for each patient to have left, bilateral, or right language dominance. The compatible value of probability of the Wada label was then acquired from these 3 values. For example, if the Wada test produced a left lateralization, then the LDP pointing to left lateralization was taken as the probability for correct classification. For each patient, the 11 analyses were ranked according to their probability of compatibility with the Wada labeling. Figure 4 upper shows the average ranking across patients of each analysis. Among the single thresholds, the strictest threshold of $0.1 \%$ produced the best ranking, while the ranking of the combination of thresholds was lower (better ranked) than each of the single thresholds alone. The Wilcoxon signed-rank test, corrected for multiple comparisons using the Holm-Bonferroni correction, showed that this effect was significant for the comparison of all
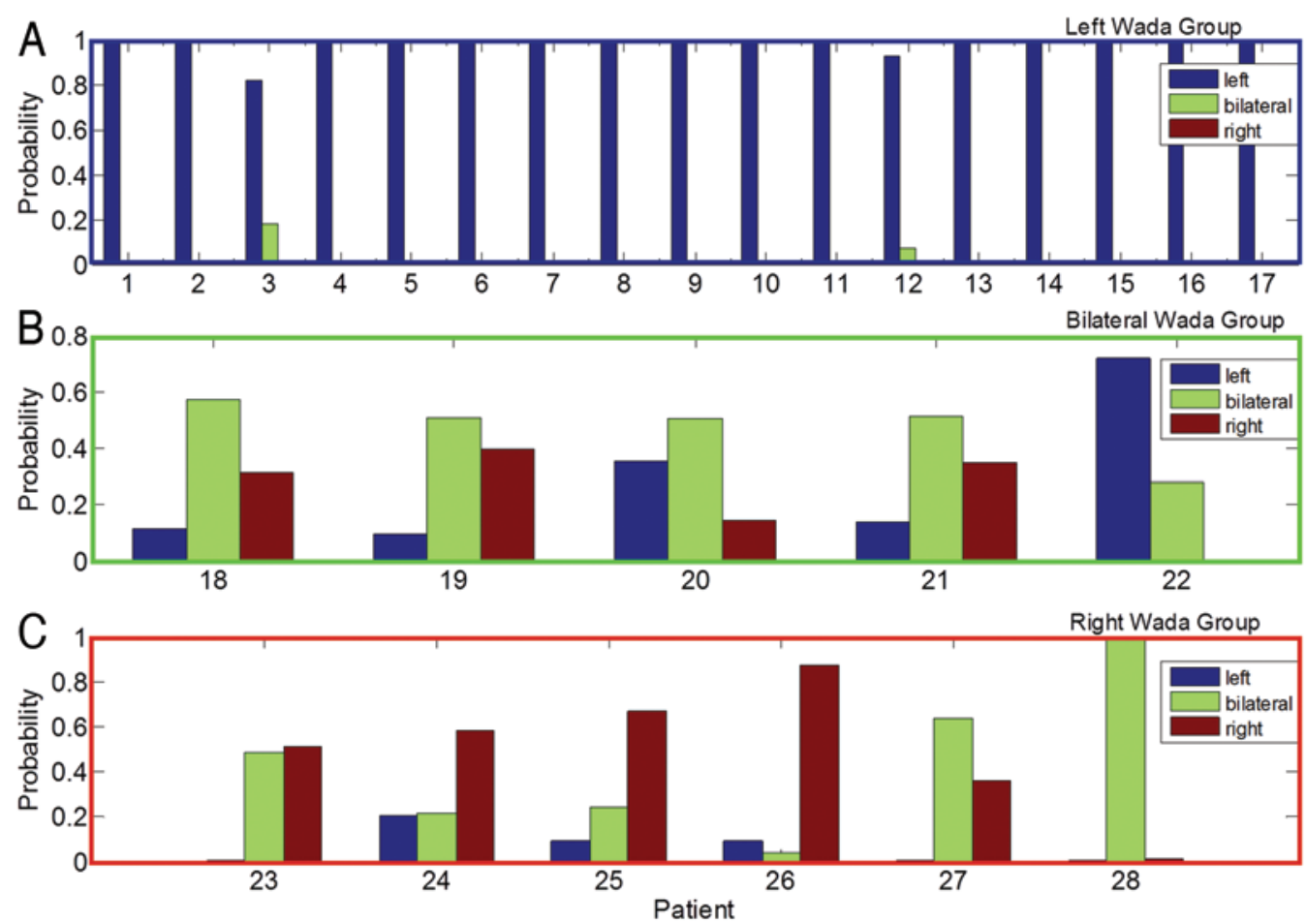

FIG. 3. Bar graphs of the final LDPs of the leave-one-out semi-supervised analysis for the 28 Wada patients using the conjunction whole-brain and multithreshold approach. The probability of each patient belonging to the left, bilateral, or right language dominant groups is shown as blue (black), green (light gray), and red (gray) bars, respectively. A: Seventeen left Wada patients. B: Five bilateral Wada patients. C: Six right Wada patients. Note that all left Wada patients were found to have the highest probability for left lateralization, 4 of 5 bilateral Wada patients were found to have the highest probability for bilateral lateralization, and 4 of 6 right Wada patients were found to have the highest probability for right lateralization. Figure is available in color online only. 

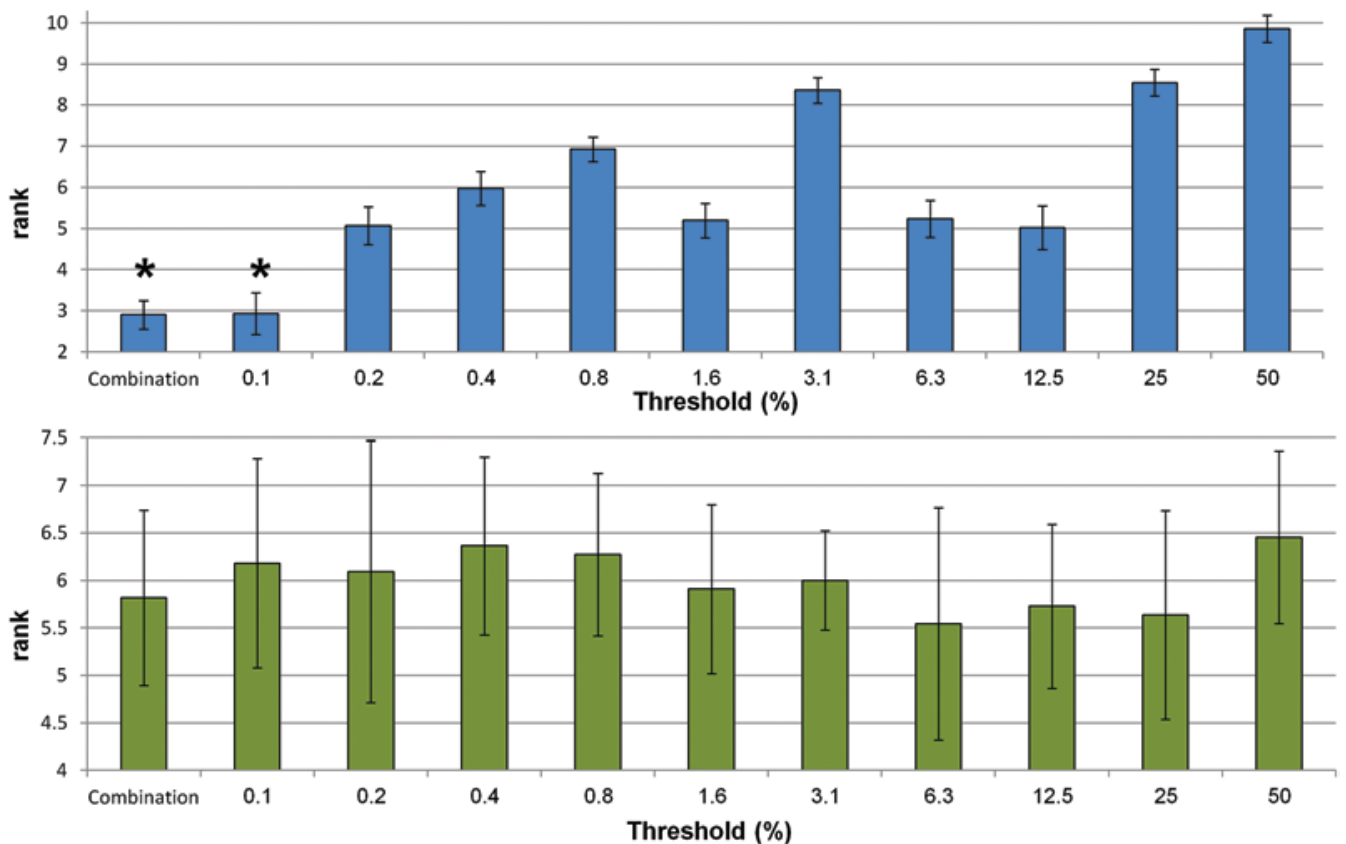

FIG. 4. Bar graphs showing comparison of multithreshold (combination) approach to each of the 10 single thresholds. Upper: For each patient, the 11 methods were ranked according to their resultant probability to correct lateralization (using the leave-one-out approach, according to the Wada result; rank 1 and 11 represents highest and lowest probability to labeling compatible with the Wada test, respectively). Bars show the mean and standard errors over 28 patients. The combination approach and the harshest threshold of $0.1 \%$ achieved significantly lower ranks (higher probability) compared with the results of the other 9 thresholds. Lower: Comparison of 11 methods for differentiating bilateral to right Wada patients. Bars show the mean and standard errors over 11 nonleft Wada patients. Figure is available in color online only.

single thresholds $(Z>2.3, p<0.05)$, with the exception of the strictest threshold $(Z=1.6, p=0.11)$. When including only bilateral and right Wada cases $(\mathrm{n}=11)$, the optimal threshold was $6.3 \%$ (Fig. 4 lower). For this group of patients, using the Wilcoxon signed-rank test to compare the combination approach to a single-threshold approach did not reveal a statistically significant difference $(\mathrm{p}>0.05)$.

\section{Multimodality Versus Single Modality}

We hypothesized that using a combination of visual and auditory verb-generation tasks would produce superior LDP results as opposed to using only a single modality (Supplementary Figure 3). We therefore performed the same logistic regression analysis on the LIs obtained from each of the 2 modalities (visual and auditory) and their conjunction. The Wilcoxon signed-rank test, corrected for 3 multiple comparisons using the Holm-Bonferroni correction, showed that the probability of obtaining a Wadacompatible label was significantly higher for the conjunction analysis compared with the visual analysis $(Z=4.46, p$ $<0.001)$ or the auditory analysis alone $(\mathrm{Z}=4.55, \mathrm{p}<0.001)$. No significant difference was found between the visual and auditory analyses $(\mathrm{Z}=0.2, \mathrm{p}=0.84)$.

\section{Supervised Versus Semi-Supervised Machine Learning}

To establish whether additional patients without Wada labeling contributed to the success of the algorithm, we compared the algorithm results of the supervised approach (including 28 patients with Wada labels) with the results of the semi-supervised approach ${ }^{3}$ (including all 76 patients). For all but 1 patient (Case 27) a higher prob- ability for Wada-compatible labeling was achieved using the semi-supervised method. A Wilcoxon signed-rank test showed that across all patients the probability for Wadacompatible labeling was significantly higher for the semisupervised logistic regression than the supervised logistic regression $(\mathrm{Z}=4.2, \mathrm{p}<0.001)$.

\section{Whole-Brain Versus Broca's Area Approach}

LDPs of 21 Wada-labeled patients were considered for Broca's area analysis (see Methods and Supplementary Fig. 2). For all but 1 patient (Case 28), a higher probability Wada-compatible lateralization was achieved using the whole-brain approach (supervised, multithreshold) compared with analyzing Broca's area only. A Wilcoxon signed-rank test showed that across all 21 patients, the probability for Wada-compatible labeling was significantly higher for the whole-brain approach than for the Broca's area approach $(\mathrm{Z}=3.96, \mathrm{p}<0.001)$.

\section{Combined Clinical, Neuropsychological, and fMRI Data Versus fMRI Data Alone}

When providing the algorithm with clinical and neuropsychological data, along with the fMRI LI scores (20 features in total, see Methods), the algorithm continues to choose the strongest weights for fMRI LI scores alone, indicating that they are the most informative for lateralization. fMRI LI scores only were used for classifying left and nonleft cases. The side of the lesion was also taken into account for differentiating right and bilateral patients. For 1 patient (Case 23), the leave-one-out algorithm used the results of the faces test in addition to fMRI LI scores 
for classification, although with a much weaker weight. Comparing final LDPs between the fMRI analysis alone and the multianalysis using fMRI, clinical, and neuropsychology data showed that the analysis of fMRI alone produced a higher probability for Wada-compatible labeling for the majority of patients. Specifically, fMRI alone outperformed the multiparametric approach in 21 of the 23 patients with neuropsychological language tests, and in 16 of 18 patients with both language and memory neuropsychological data (Cases 3 and 23 were exceptions). A Wilcoxon signed-rank test showed that the probability for Wada-compatible lateralization was significantly higher for the fMRI data alone than the combination of fMRI, clinical, and neuropsychological data $(\mathrm{Z}=3.34, \mathrm{p}<0.001$ for fMRI data alone vs neuropsychological language data only and $\mathrm{Z}=3.05, \mathrm{p}<0.05$ for fMRI data alone vs both language and memory neuropsychological data).

\section{LDP Versus Standard Cutoff Procedure}

Applying the method used by Janecek et al..$^{22}$ to our data, we found that 16 of the 17 left-dominant Wada patients and all right-dominant Wada patients would have been classified correctly (Wada compatible); however, none of the 5 bilateral Wada cases would have been correctly (Wada compatible) classified. As our results show a tendency for this group to have negative LI values, they would have all been classified as right lateralized. Thus, using the classical cutoff method, 22 patients out of the 28 patients were classified in accordance with their Wada result (78\%).

\section{Discussion}

We present a new, automatic method for providing neurosurgical candidates and their clinicians with the probability for language lateralization. Of the 28 patients who underwent a Wada examination (17 left, 5 bilateral, and 6 right dominance according to the Wada test), 25 patients exhibited the highest probability for left, right, or bilateral dominance that was consistent with the lateralization provided by this invasive method. This represents a success rate of $89.3 \%$ in correspondence to the Wada test. Interestingly, 4 of the 5 bilateral patients showed more activation in the right hemisphere making the classification between bilateral and right classes a relatively difficult task. This corresponds to previous reports in the literature that have grouped the bilateral and right patients into 1 group (the "atypical" lateralization group ${ }^{44}$ ). The algorithm used in the present study was thus $96 \%$ successful in classifying left-dominant patients and further differentiating them from the other 2 groups (bilateral and right-dominant patients). Moreover, the algorithm was $82 \%$ successful in differentiating bilateral and right-dominant patients, with only 2 right-dominant Wada patients exhibiting a highest probability for bilateral dominance using fMRI. Thus, despite the fact that the right and bilateral patient groups have close lateralization profiles, it is still possible to further classify them, providing informative probabilities for language dominance. In contrast, the standard state-ofthe-art laterality classification algorithm ${ }^{22}$ indicated less than chance performance (5 of 11 Wada-compatible classifications) for this atypical group. It must be taken into consideration that the algorithm's success rates presented here are based on the Wada examination being used as a "ground truth" comparison. Since the Wada test may also produce erroneous lateralization in a minority of cases, ${ }^{27,30}$ the true success rates for lateralization may be slightly better or worse than those presented.

Producing a probabilistic output for individual patient language laterality is one of the main advances of this study. Both clinical teams and patients are specifically concerned about the probability of surgery resulting in language deficits. Providing them with the probability of language lateralization does not directly address this concern, since cognitive deficiencies following surgery are a function of additional variables, such as the location and extent of resection, vascular damage during surgery, and the quality of intraoperative mapping. However, the probabilistic output of the method described in this work takes the clinicians a step further toward assessing this risk, and in our experience this step is of great value for both the neurosurgeon and the patient.

To date, the common practice in presurgical mapping involves a qualitative assessment of language dominance based on fMRI activation maps. Though a number of techniques have been suggested for a quantitative classification of lateralization groups, they have not been widely used in clinical settings. Some studies have classified patients into different groups using a cutoff threshold of the lateralization value. ${ }^{6,37}$ Because different thresholds may produce opposite lateralization results, ${ }^{23}$ the threshold problem has become a significant issue in the literature, ${ }^{30}$ and different thresholds have been applied. In the present study, we adopted the algorithm used by Janecek et al., ${ }^{22}$ the most comprehensive study comparing Wada and fMRI results (229 patients), to our lateralization data. However, this algorithm provided unsatisfactory results (none of our 5 bilateral Wada cases were classified in accordance with the Wada test result). Thus, the advantage of the machinelearning method proposed here is 2-fold: it allows the algorithm to choose the most informative thresholds in a modular fashion (different thresholds can be chosen to differentiate between the different laterality groups), and it provides a probability instead of a strict classification.

In an attempt to characterize optimal LDP methodologies, the current study tested 5 main inquiries including 1) multiple versus single thresholds, 2) semi-supervised versus supervised learning, 3) multiple versus single modalities, 4) whole-brain versus regional analysis, and 5) the advantage of combining clinical and neuropsychological information with fMRI LIs. Given the limitations of the Wada test as a gold standard, ${ }^{27}$ the results of our analysis showed the following: 1) performing machine learning on a combination of multiple thresholds produces better results than machine learning that is based on each single threshold (moreover, multithreshold analysis has the ability to detect the best thresholds for each of the 2 classifications: when classifying between typical [left] and nontypical [right or bilateral] dominant patients, it is preferable to use only brain regions showing the strongest activations, and when classifying within the atypical group [right vs bilateral] the use of a larger brain network is required); 2) combining visual and auditory fMRI data outperformed 
single-modality analysis (visual or auditory alone); 3) the inclusion of patients without invasive Wada labeling (semi-supervised learning) added substantial information over the labeled data set alone (supervised learning), as 27 of the 28 patients had a higher probability of classification that matched the Wada test results when including information provided by the non-Wada group; 4) a wholebrain approach was found to be superior to the regional approach, using Broca's area only; and 5) clinical data and neuropsychological testing did not increase the success rate of the algorithm, at least not for the study group presented here, and the fMRI results were found to be sufficient for probabilistic classification.

Despite the promising results found in the current study, several limitations must be considered. In the analysis presented, the results of the Wada test are considered the gold standard for language lateralization; however, several studies have shown that the Wada test can be misleading in a minority of patients. ${ }^{27,30}$ Future work may include postoperative neuropsychological testing as well as intraoperative language mapping to more firmly establish the laterality used as a gold standard. Additionally, the number of bilateral and right-dominant patients ascertained from the Wada test is quite small; thus future work should increase this atypical dominance group to better establish lateralization, possibly by adding patients with alternative invasive validations.

\section{Conclusions}

The output of the current study presents an automatic method for calculating the probabilities of any patient as having left, bilateral, or right language dominance prior to surgery. Compatibility with invasive lateralization was achieved for $89 \%$ of patients. The bilateral group showed fMRI activation profiles more similar to the right-dominant group than to the left-dominant group. The inclusion of patients without invasive Wada labeling added substantial information over the analysis using only the labeled data set. This is one of the few reports to provide the probability for language lateralization of patients with epilepsy based on fMRI, and the first report that we know of to use semi-supervised learning to investigate this matter. Accuracy improved by enlarging the group of patients the algorithm uses for learning by complementing the results obtained in Wada-lateralized patients with those obtained in patients who did not undergo invasive lateralization. It is also one of the few reports, if any, using different thresholds to classify the different laterality groups. No predetermined parameters are necessary, such as an a priori determination of threshold. This work provides an initial database of patients with known lateralization ascertained from invasive procedures, and such future patients will help to further enrich this database, thus advancing toward improved classification accuracy for all patients to follow. The presented technique is not limited to language lateralization and can be directly implemented to other cognitive processes such as memory, attention, and reasoning. We hope that this work and future implementations of it will be of significant value in providing a quantitative and objective estimation of lateralization of cognitive process- es for both the clinical and scientific communities. The software described in this paper can be obtained at http:/ fmri-tlv.org.

\section{Acknowledgments}

We would like to thank Uri Shalit for the advice and discussions on issues regarding machine learning and Maya Bleich-Cohen for discussions on cognitive issues. This research has been supported by a European Union ACTIVE grant (FP7-ICT-2009-6-270460) and the Sagol Center for Brain Informatics, TASMC.

\section{References}

1. Abbott DF, Waites AB, Lillywhite LM, Jackson GD: fMRI assessment of language lateralization: an objective approach. Neuroimage 50:1446-1455, 2010

2. Adcock JE, Wise RG, Oxbury JM, Oxbury SM, Matthews PM: Quantitative fMRI assessment of the differences in lateralization of language-related brain activation in patients with temporal lobe epilepsy. Neuroimage 18:423-438, 2003

3. Amini MR, Gallinari P: Semi-supervised logistic regression, in 15th European Conference on Artificial Intelligence. Amsterdam: IOS Press, 2002, pp 390-394

4. Andelman F, Kipervasser S, Maimon S, Fried I, Parmet Y, Neufeld MY: A revised intracarotid etomidate memory (Wada) procedure. Acta Neurol Scand 127:97-102, 2013

5. Andelman F, Kipervasser S, Reider-Groswasser II, Fried I, Neufeld MY: Hippocampal memory function as reflected by the intracarotid sodium methohexital Wada test. Epilepsy Behav 9:579-586, 2006

6. Arora J, Pugh K, Westerveld M, Spencer S, Spencer DD, Todd Constable R: Language lateralization in epilepsy patients: fMRI validated with the Wada procedure. Epilepsia 50:2225-2241, 2009

7. Baxendale S: The Wada test. Curr Opin Neurol 22:185-189, 2009

8. Binder JR, Swanson SJ, Hammeke TA, Morris GL, Mueller WM, Fischer M, et al: Determination of language dominance using functional MRI: a comparison with the Wada test. Neurology 46:978-984, 1996

9. Bishop CM: Pattern Recognition and Machine Learning. New York: Springer, 2006

10. Bleich-Cohen M, Sharon H, Weizman R, Poyurovsky M, Faragian S, Hendler T: Diminished language lateralization in schizophrenia corresponds to impaired inter-hemispheric functional connectivity. Schizophr Res 134:131-136, 2012

11. Carpentier A, Pugh KR, Westerveld M, Studholme C, Skrinjar O, Thompson JL, et al: Functional MRI of language processing: dependence on input modality and temporal lobe epilepsy. Epilepsia 42:1241-1254, 2001

12. Chapelle O, Scholkopf B, Zien A: Semi-Supervised Learning. Cambridge, MA: MIT Press, 2006

13. Chlebus P, Mikl M, Brázdil M, Pazourková M, Krupa P, Rektor I: fMRI evaluation of hemispheric language dominance using various methods of laterality index calculation. Exp Brain Res 179:365-374, 2007

14. Cooper GF, Aliferis CF, Ambrosino R, Aronis J, Buchanan BG, Caruana R, et al: An evaluation of machine-learning methods for predicting pneumonia mortality. Artif Intell Med 9:107-138, 1997

15. D'Esposito M: Functional neuroimaging of cognition. Semin Neurol 20:487-498, 2000

16. Deblaere K, Boon PA, Vandemaele P, Tieleman A, Vonck K, Vingerhoets G, et al: MRI language dominance assessment in epilepsy patients at $1.0 \mathrm{~T}$ : region of interest analysis and comparison with intracarotid amytal testing. Neuroradiology 46:413-420, 2004 
17. Desmond JE, Sum JM, Wagner AD, Demb JB, Shear PK, Glover GH, et al: Functional MRI measurement of language lateralization in Wada-tested patients. Brain 118:1411-1419, 1995

18. Dion JE, Gates PC, Fox AJ, Barnett HJ, Blom RJ: Clinical events following neuroangiography: a prospective study. Stroke 18:997-1004, 1987

19. Dreiseitl S, Ohno-Machado L, Kittler H, Vinterbo S, Billhardt $\mathrm{H}$, Binder $\mathrm{M}$ : A comparison of machine learning methods for the diagnosis of pigmented skin lesions. J Biomed Inform 34:28-36, 2001

20. Dym RJ, Burns J, Freeman K, Lipton ML: Is functional MR imaging assessment of hemispheric language dominance as good as the Wada test?: a meta-analysis. Radiology 261:446-455, 2011

21. Helmstaedter C, Kurthen M, Linke DB, Elger CE: Patterns of language dominance in focal left and right hemisphere epilepsies: relation to MRI findings, EEG, sex, and age at onset of epilepsy. Brain Cogn 33:135-150, 1997

22. Janecek JK, Swanson SJ, Sabsevitz DS, Hammeke TA, Raghavan M, Rozman M, et al: Language lateralization by fMRI and Wada testing in 229 patients with epilepsy: rates and predictors of discordance. Epilepsia 54:314-322, 2013

23. Jones SE, Mahmoud SY, Phillips MD: A practical clinical method to quantify language lateralization in fMRI using whole-brain analysis. Neuroimage 54:2937-2949, 2011

24. Kavé G: Phonemic fluency, semantic fluency, and difference scores: normative data for adult Hebrew speakers. J Clin Exp Neuropsychol 27:690-699, 2005

25. Kavé G: Standardization and norms for a Hebrew naming test. Brain Lang 92:204-211, 2005

26. Kearns M, Ron D: Algorithmic stability and sanity-check bounds for leave-one-out cross-validation. Neural Comput 11:1427-1453, 1999

27. Kirsch HE, Walker JA, Winstanley FS, Hendrickson R, Wong STC, Barbaro NM, et al: Limitations of Wada memory asymmetry as a predictor of outcomes after temporal lobectomy. Neurology 65:676-680, 2005

28. Lehéricy S, Cohen L, Bazin B, Samson S, Giacomini E, Rougetet R, et al: Functional MR evaluation of temporal and frontal language dominance compared with the Wada test. Neurology 54:1625-1633, 2000

29. Maldjian JA, Laurienti PJ, Kraft RA, Burdette JH: An automated method for neuroanatomic and cytoarchitectonic atlas-based interrogation of fMRI data sets. Neuroimage 19:1233-1239, 2003

30. Meador KJ, Loring DW: The Wada test: controversies, concerns, and insights. Neurology 52:1535-1536, 1999

31. Pillai JJ, Zaca D: Relative utility for hemispheric lateralization of different clinical fMRI activation tasks within a comprehensive language paradigm battery in brain tumor patients as assessed by both threshold-dependent and threshold-independent analysis methods. Neuroimage 54 (Suppl 1):S136-S145, 2011

32. Powell HW, Parker GJ, Alexander DC, Symms MR, Boulby PA, Barker GJ, et al: Imaging language pathways predicts postoperative naming deficits. J Neurol Neurosurg Psychiatry 79:327-330, 2008

33. Price CJ: The anatomy of language: a review of $100 \mathrm{fMRI}$ studies published in 2009. Ann N Y Acad Sci 1191:62-88, 2010

34. Ramsey NF, Sommer IE, Rutten GJ, Kahn RS: Combined analysis of language tasks in fMRI improves assessment of hemispheric dominance for language functions in individual subjects. Neuroimage 13:719-733, 2001

35. Roux FE, Boulanouar K, Lotterie JA, Mejdoubi M, LeSage JP, Berry I: Language functional magnetic resonance imaging in preoperative assessment of language areas: correlation with direct cortical stimulation. Neurosurgery 52:13351347,2003

36. Rutten GJ, Ramsey NF, van Rijen PC, Noordmans HJ, van
Veelen CW: Development of a functional magnetic resonance imaging protocol for intraoperative localization of critical temporoparietal language areas. Ann Neurol 51:350-360, 2002

37. Sabbah P, Chassoux F, Leveque C, Landre E, Baudoin-Chial $\mathrm{S}$, Devaux B, et al: Functional MR imaging in assessment of language dominance in epileptic patients. Neuroimage 18:460-467, 2003

38. Sabsevitz DS, Swanson SJ, Hammeke TA, Spanaki MV, Possing ET, Morris GL III, et al: Use of preoperative functional neuroimaging to predict language deficits from epilepsy surgery. Neurology 60:1788-1792, 2003

39. Schmidt M: Rey Auditory Verbal Learning Test: A Handbook. Los Angeles: Western Psychological Services, 1996

40. Springer JA, Binder JR, Hammeke TA, Swanson SJ, Frost JA, Bellgowan PS, et al: Language dominance in neurologically normal and epilepsy subjects: a functional MRI study. Brain 122:2033-2046, 1999

41. van 't Veer, LJ, Dai H, Van De Vijver MJ, He YD, Hart AA, Mao M, et al: Gene expression profiling predicts clinical outcome of breast cancer. Nature 415: 530-536, 2002

42. Wada J, Rasmussen T: Intracarotid injection of sodium amytal for the lateralization of cerebral speech dominance: experimental and clinical observations. J Neurosurg 17:266-282, 1960

43. Wechsler D: WMS-III: Wechsler Memory Scale Administration and Scoring Manual. San Antonio: The Psychological Corporation, 1997

44. Woermann FG, Jokeit H, Luerding R, Freitag H, Schulz R, Guertler $\mathrm{S}$, et al: Language lateralization by Wada test and fMRI in 100 patients with epilepsy. Neurology 61:699-701, 2003

45. Wood AG, Harvey AS, Wellard RM, Abbott DF, Anderson $\mathrm{V}$, Kean M, et al: Language cortex activation in normal children. Neurology 63:1035-1044, 2004

\section{Disclosures}

The authors report no conflict of interest concerning the materials or methods used in this study or the findings specified in this paper.

\section{Author Contributions}

Conception and design: Gazit, Glikmann-Johnston, Hendler, Perry. Acquisition of data: Gazit, Andelman, Glikmann-Johnston, Shapira-Lichter, Ovadia, Kipervasser, Neufeld, Fried, Hendler, Perry. Analysis and interpretation of data: Gazit, Andelman,ShapiraLichter. Drafting the article: Andelman, Glikmann-Johnston, Solski, Shapira-Lichter, Kipervasser, Perry. Critically revising the article: Gazit, Andelman, Glikmann-Johnston, Gonen, Perry. Reviewed submitted version of manuscript: Solski, Perry. Statistical analysis: Gazit, Gonen. Study supervision: Hendler.

\section{Supplemental Information}

\section{Online-Only Content}

Supplemental material is available with the online version of the article.

Appendix and Supplementary Figures 1-3. http://thejns.org/ doi/suppl/10.3171/2015.7.JNS142568.

\section{Current Affiliations}

Dr. Glikmann-Johnston: School of Psychological Sciences, Faculty of Medicine, Nursing and Health Sciences, Monash University, Victoria, Australia.

\section{Correspondence}

Tomer Gazit, Tel Aviv Medical Center, 6 Weizman St., Tel Aviv 6423906, Israel. email: tomergazit@gmail.com. 